\title{
Haag-Ruelle Approximation of Collision States
}

\author{
Detlev Buchholz \\ II. Institut für Theoretische Physik der Universität Hamburg, \\ Hamburg, Federal Republic of Germany \\ Received February 8, 1974
}

\begin{abstract}
We investigate the rate of convergence of the Haag-Ruelle approximation $\Psi(t)$ at large times $t$ for arbitrary collision states $\Psi$ with finite energy. An improved estimate of the norm distance $\|\Psi-\Psi(t)\|$ is given. In particular for states $\Psi$ with smooth asymptotic wave functions it turns out that $\|\Psi-\Psi(t)\|$ approaches 0 almost like $t^{-3 / 4}$.
\end{abstract}

\section{Introduction}

The fundamental work of Haag [1] and Ruelle [2] established the existence of states which can be interpreted as asymptotic particle configurations within the framework of quantum field theory. Since this ingenious construction is by now well known it may suffice to sketch the procedure briefly: given any incoming or outgoing particle configuration one can construct sequences of vectors $\Psi(t)$ in a Hilbert space $\mathscr{H}$ by applying products of suitably chosen almost local oneparticle creation operators at time $t$ to the vacuum vector. These HaagRuelle approximations converge strongly in the limit of large negative and positive times and the limit vectors $\Psi^{\text {in }}$ and $\Psi^{\text {out }}$ correspond to the given incoming and outgoing particle configuration respectively.

One might think that one can forget about the approximations $\Psi(t)$ once one has constructed the collision states $\Psi^{\text {in }}, \Psi^{\text {out }}$ since all the information relevant for physics is contained in matrix elements which are computable from these vectors. However, for some problems it is sufficient and much simpler to consider the approximations of a given collision state instead of the state itself. Several interesting results in collision theory have been derived from the well known kinematical properties of the vectors $\Psi(t)$ at finite times $t$ and the convergence behaviour of the sequences $\Psi(t)$. For example, Araki and Haag showed that local observables provide a direct interpretation of scattering states as asymptotic particle configurations [3]. For a detailed summary of results of more technical nature see the lecture notes of Araki [4] and Hepp [5].

In all these investigations, the crucial point is to derive an adequate estimate for the norm distances $\left\|\Psi^{\text {in }}-\Psi(t)\right\|$ and $\left\|\Psi^{\text {out }}-\Psi(t)\right\|$ at large 
negative and positive times $t$ respectively. In the papers quoted above such estimates are given for asymptotic configurations which are well behaved: technically speaking, the wavefunctions corresponding to the asymptotic particle configurations are supposed to be elements of the Schwartz-space $\mathscr{S}$. Unfortunately, such configurations are not the only important ones in collision theory. Under the influence of interaction even those states with a smooth incoming wavefunction can have an outgoing configuration which, in many cases, can no longer be described by a function from $\mathscr{S}$. Thus one is forced to consider wavefunctions which are not continuously differentiable if, for example, one is interested in the spatio-temporal behaviour of collision states. For this reason we investigate the convergence behaviour of the HaagRuelle approximations $\Psi(t)$ for arbitrary asymptotic configurations.

In this paper we give an estimate which clarifies the connection between the degree of singularity of the wavefunctions in momentum space and the decrease of the norm distances $\left\|\Psi^{\text {in }}-\Psi(t)\right\|$ and $\left\|\Psi^{\text {out }}-\Psi(t)\right\|$ for asymptotic $t$. Moreover, for smooth wavefunctions our estimate is an improvement on previous known ones: it turns out that $\left\|\Psi^{\text {out }}-\Psi(t)\right\|$ approaches 0 almost like $t^{-3 / 4}$ for large $t$. (An analogous statement holds for the states $\Psi^{\text {in }}$.) This is only a slight modification of the asymptotic $t^{-1 / 2}$ behaviour which was proved by Haag and Ruelle [1], [2]. Yet we shall show in a forthcoming paper that our result is the optimal one to be expected within the general framework of quantum field theory. There should be models compatible with the basic postulates in which our result can not be improved.

Our methods of proof can easily be carried over to the case of nonrelativistic potential scattering theory for short range potentials. We want also to point out that our arguments can be applied in a modelworld of arbitrary spatial dimension. We do not need to assume that the dimension of space is greater than 2 .

\section{Assumptions and Definitions}

Our arguments do not depend very sensitively on the framework in which the basic postulates of quantum field theory are expressed. Since we want to avoid all unnecessary complications we shall formulate our assumptions in terms of the field-algebra ${ }^{1}$ instead of the fieldoperators (Wightman-fields) themselves. Thus we shall never have to worry about domain questions etc. Yet at the expense of increased technicality we could have derived our results in the Wightman-frame-

1 Roughly speaking, the field algebra is generated by all bounded functions of the selfadjoint components of the fields. 
work [6] as well. For the sake of simplicity we shall furthermore restrict our attention to a theory with only one kind of particle which we shall assume to be a massive neutral boson with spin 0 .

With these simplifications, the Hilbert space $\mathscr{H}$ of all physical states is a direct sum of the 1-dimensional space $\{c \cdot \Omega\}$ corresponding to the vacuum, the one particle space $\mathscr{H}_{1}$ and the space of the collision states. We assume furthermore that $\mathscr{H}$ carries a continuous unitary representation of the translations $x=(t, x) \rightarrow U(x)$ and that the vacuum $\Omega$ is the only vector invariant under the action of $U(x)$. In this paper we neither make explicitly use of the Lorentz-covariance nor of the local properties of the field algebras nor of the positivity of the energy. (For a review of the basic postulates see [4].) But we shall use some properties of a relativistic quantum theory which are consequences of these fundamental assumptions. It is crucial for our argument that there exists a set $\mathscr{P}$ of almost local 1-particle creation operators with properties specified in the following theorem [3], [7]:

Theorem. For each compact set $\mathbb{K} \subset \mathbb{R}^{3}$ there exists a bounded operator $A \in \mathscr{P}$ such that

i) $A \Omega$ is a one particle state, $A \Omega \in \mathscr{H}_{1}$, and $A^{*} \Omega=0$.

ii) the 1-particle wavefunction of $A \Omega$ in momentum space is constant on $\mathbb{K}$, more precisely:

$$
(p, A \Omega)=(2 \pi)^{-3 / 2} \quad \text { for } \quad p \in \mathbb{K} .
$$

iii) the Fourier transform $\tilde{A}(p)$ of $A(x)=U(x) A U^{-1}(x)$ has compact support (in the sense of distributions). As a consequence, the derivatives of $A(x)$ with respect to the translations exist in the norm topology.

iv) $A$ together with its derivatives is almost local. By this we mean the following: let $A_{1}, \ldots, A_{n}$ stand for $A$, a derivative of $A$ or their adjoints. Then the truncated vacuum expectation value of the translated operators has the following decrease property:

$$
\left|\left(\Omega, A_{1}\left(\boldsymbol{x}_{1}\right) \ldots A_{n}\left(\boldsymbol{x}_{n}\right) \Omega\right)_{T}\right| \leqq \varphi\left(\sum_{i<j}\left|\boldsymbol{x}_{i}-\boldsymbol{x}_{j}\right|\right) .
$$

$\varphi$ is a continuous, monotone function which decreases faster than any inverse power of its argument, $\lim _{v \rightarrow \infty} v^{N} \varphi(v)=0$ for all $N \in \mathbb{N}$.

The set $\mathscr{P}$ will be used in the following to construct the collision states: let $\Psi_{f_{n}}^{\text {out }}$ be a vector in $\mathscr{H}$ corresponding to an outgoing $n$-particle configuration with a momentum distribution $\tilde{f}_{n}\left(\boldsymbol{p}_{1} \ldots \boldsymbol{p}_{n}\right) .\left(\Psi_{f_{n}}^{\mathrm{in}}\right.$ is defined analogously.) If $\tilde{f}_{n}\left(\boldsymbol{p}_{1} \ldots \boldsymbol{p}_{n}\right)$ has compact support in $\mathbb{R}^{3 n}$ we can find a compact set $\mathbb{K} \subset \mathbb{R}^{3}$ such that $\operatorname{supp} \tilde{f}_{n} \subset \mathbb{K}^{n}$. ( $\mathbb{K}^{n}$ is the $n$-fold Cartesian product of $\mathbb{K}$.) Corresponding to $\mathbb{K}$ we take an operator $A$ from $\mathscr{P}$ 
with properties listed in the theorem and construct the Haag-Ruelle approximations of $\Psi_{f_{n}}^{\text {out }}$,

$$
\Psi_{f_{n}}(t)=\int \prod_{l=1}^{n} d^{3} x_{l} f_{n}\left(t \mid \boldsymbol{x}_{1} \ldots \boldsymbol{x}_{n}\right) A\left(t, \boldsymbol{x}_{1}\right) \ldots A\left(t, \boldsymbol{x}_{n}\right) \Omega .
$$

Here we have introduced the configuration space wavefunction at time $t$,

$$
f_{n}\left(t \mid \boldsymbol{x}_{1} \ldots \boldsymbol{x}_{n}\right)=(2 \pi)^{-3 n / 2} \int \prod_{l=1}^{n} d^{3} p_{l} e^{-i t \omega_{l}+i \boldsymbol{x}_{l} \boldsymbol{p}_{l}} \cdot \tilde{f}_{n}\left(\boldsymbol{p}_{1} \ldots \boldsymbol{p}_{n}\right)
$$

using the abbreviation $\omega_{l}=\omega_{\boldsymbol{p}_{l}}=\left(\left|\boldsymbol{p}_{l}\right|^{2}+\mu^{2}\right)^{1 / 2} ; \mu$ is the mass of the particle. As we remarked in the introduction, the work of Haag and Ruelle implies that the sequence $\Psi_{f_{n}}(t)$ converges strongly towards $\Psi_{f_{n}}^{\text {out }}$ and that $\left\|\Psi_{f_{n}}^{\text {out }}-\Psi_{f_{n}}(t)\right\| \leqq c \cdot t^{-1 / 2}$ for large positive $t$ if the wavefunction $\tilde{f}_{n}\left(\boldsymbol{p}_{1} \ldots \boldsymbol{p}_{n}\right)$ is smooth. For a special class of configurations which are characterized by smooth wavefunctions $f_{n}$ with "non-overlapping momenta" ${ }^{2}$ Hepp [5] and independently Araki and Haag [3] could give a much better estimate: for $t>0$ and any $N \in \mathbb{N}$

$$
\left\|\Psi_{f_{n}}^{\text {out }}-\Psi_{f_{n}}(t)\right\| \leqq c_{N} \cdot t^{-N}
$$

This observation will be the starting point for our work.

\section{Approximation of Arbitrary Collision States}

We shall show now how the Haag-Ruelle approximation $\Psi_{f}(t)$ converges if $f^{3}$ is an arbitrary square-integrable wavefunction with compact support in momentum space. The underlying idea of proof is very simple: we split the function $f$ into the sum of a smooth function $g$ with non-overlapping momenta and the (possibly) singular remainder $\Delta f=f-g$. According to Eq. (1) in the preceding chapter we get then a decomposition of $\Psi_{f}(t): \Psi_{f}(t)=\Psi_{g}(t)+\Psi_{\Delta f}(t)$. Now $\Psi_{g}(t)$ converges very rapidly towards $\Psi_{g}^{\text {out }}$ for large $t$ (see relation (3) above) and $\Psi_{\Delta f}(t)$, $\Psi_{\Delta f}^{\text {out }}$ both have small norms if $g$ is a reasonable approximation of $f$. So one has only to find an appropriate decomposition of $f$ at each time $t$ in order to get a good estimate for the rate of convergence of $\left\|\Psi_{f}^{\text {out }}-\Psi_{f}(t)\right\|$.

For a quantitative result we need an estimate of the asymptotic behaviour of $\Psi_{g}(t)$ which is better than the one given by inequality (3). We have to control how the constant $c_{N}$ in this relation depends on the properties of the function $g$. For this purpose we introduce a set of

2 The function $f_{n}$ is said to have "non-overlapping momenta" if the support of $\tilde{f}_{n}\left(\boldsymbol{p}_{1} \ldots \boldsymbol{p}_{n}\right)$ has a finite distance from the planes $\boldsymbol{p}_{i}-\boldsymbol{p}_{j}=0, i \neq j$ in $\mathbb{R}^{3 n}, n \geqq 2$.

${ }^{3}$ Here we have omitted the index $n$ of $f$ since we shall consider throughout this chapter only $n$-particle states and their approximations. 
norms on the linear subspace $\mathscr{\mathscr { S }}\left(\mathbb{R}^{3 n}\right)$ of $\mathscr{S}\left(\mathbb{R}^{3 n}\right)$ which is spanned by the functions with non-overlapping momenta.

Definition. Let $g$ be an element of $\mathscr{\mathscr { S }}\left(\mathbb{R}^{3 n}\right)$. Then for each $N \in \mathbb{N}$ we define a norm $\|g\|_{N}$ of $g$ by

$$
\begin{aligned}
\|g\|_{N}^{2}=\sum_{i+|j| \leqq N} \int & \prod_{l=1}^{n} d^{3} p_{l} \mid\left(1+\sum_{l^{\prime}=1}^{n}\left|\boldsymbol{p}_{l^{\prime}}\right|\right)^{3 N} \\
& \left.\cdot\left(1+\sum_{m<m^{\prime}} \frac{1}{\left|\boldsymbol{p}_{m}-\boldsymbol{p}_{m^{\prime}}\right|}\right)^{N+i} \cdot \tilde{g}^{(j)}\left(\boldsymbol{p}_{1} \ldots \boldsymbol{p}_{n}\right)\right|^{2} .
\end{aligned}
$$

Here we have introduced the multi-index $(j)=j_{1} \ldots j_{3 n} ;|j|=\sum_{l=1}^{3 n} j_{l}$ and

$$
\tilde{g}^{(j)}\left(\boldsymbol{p}_{1} \ldots \boldsymbol{p}_{n}\right)=\frac{\partial^{j^{1}}}{\partial\left(p_{1}\right)_{1}^{j^{1}}} \ldots \frac{\partial^{j_{3 n}}}{\partial\left(p_{n}\right)_{3}^{j_{3 n}}} \tilde{g}\left(\boldsymbol{p}_{1} \ldots \boldsymbol{p}_{n}\right) .
$$

Besides the norms $\|g\|_{N}$ we shall also consider the norm $\|g\|$ of $g$ in $L^{2}\left(\mathbb{R}^{3 n}\right)$,

$$
\|g\|^{2}=\int \prod_{l=1}^{n} d^{3} p_{l} \cdot\left|\tilde{g}\left(\boldsymbol{p}_{1} \ldots \boldsymbol{p}_{n}\right)\right|^{2} .
$$

With this notation we are now able to state our main result:

Theorem. Let $f$ be an element of $L^{2}\left(\mathbb{R}^{3 n}\right)$ with support in a given compact region $\mathbb{K}^{n}$ of momentum space ${ }^{4}$. Then the following inequality holds for arbitrary elements $g \in \mathscr{S}^{\circ}\left(\mathbb{R}^{3 n}\right)$ and $N \in \mathbb{N}, N>1$ :

$$
\left\|\Psi_{f}^{\text {out }}-\Psi_{f}(t)\right\| \leqq c_{N} \cdot\|g\|_{N} \cdot t^{-N+1}+c \cdot\|f-g\| \text { for } t>0 .
$$

The constants $c_{N}$ and $c$ neither depend on $f$ nor on $g$ but they may depend on the size and location of $\mathbb{K}$. (An analogous result holds for $\Psi_{f}^{\text {in }}$.)

Before we start to prove this theorem let us discuss some consequences. It is obvious from the theorem and from the definition of the norms $\|.\|_{N}$ that there are two independent sources for a slow decrease of the norm distance $\left\|\Psi_{f}^{\text {out }}-\Psi_{f}(t)\right\|$ for large $t$ : a bad momentum space behaviour and possible threshold contributions (at the points $\boldsymbol{p}_{i}=\boldsymbol{p}_{j}$, $i \neq j$ ) of the wavefunction $f$. Corresponding to this fact one splits up the function $f$ into a singular and a non-singular part and then decomposes the non-singular part into a threshold contribution and a function with non-overlapping momenta. The last term can then be taken as a suitable approximation of $f$.

The first step of this procedure may be performed by a convolution of $f$ with smooth approximations of the $\delta$-function: let $\varphi$ be any element

${ }_{4}$ We do not need to mention explicitly that $\tilde{f}\left(\boldsymbol{p}_{1} \ldots \boldsymbol{p}_{n}\right)$ must be totally symmetric in $p_{1} \ldots p_{n}$ since it is an asymptotic wavefunction. 
of $\mathscr{S}\left(\mathbb{R}^{3 n}\right)$ with compact support in momentum space and satisfying the normalisation condition $\int \prod_{l=1}^{n} d^{3} p_{l} \tilde{\varphi}\left(\boldsymbol{p}_{1} \ldots \boldsymbol{p}_{n}\right)=1$. Then the functions $(s \geqq 1)$

$$
\tilde{f}_{s}\left(\boldsymbol{p}_{1} \ldots \boldsymbol{p}_{n}\right)=\int \prod_{l=1}^{n} d^{3} q_{l} \cdot s^{3 n} \tilde{\varphi}\left(s\left[\boldsymbol{p}_{1}-\boldsymbol{q}_{1}\right] \ldots s\left[\boldsymbol{p}_{n}-\boldsymbol{q}_{n}\right]\right) \cdot \tilde{f}\left(\boldsymbol{q}_{1} \ldots \boldsymbol{q}_{n}\right)
$$

are elements of $\mathscr{S}\left(\mathbb{R}^{3 n}\right)$ with support within a fixed compact region of momentum space. It is easy to verify that the norms of the derivatives $\tilde{f}_{s}^{(j)}\left(\boldsymbol{p}_{1} \ldots \boldsymbol{p}_{n}\right)$ in $L^{2}\left(\mathbb{R}^{3 n}\right)$ have the bounds $\left\|f_{s}^{(j)}\right\| \leqq c_{|j|} \cdot\left(1+s^{|j|}\right)$ and that $\left\|f-f_{s}\right\|$ approaches 0 if $s$ becomes large. We divide the wavefunctions $f$ into classes with the same degree of singularity in momentum space by looking at the rate of convergence of $\left\|f-f_{s}\right\|$.

Definition. The linear space $M_{\alpha}\left(\mathbb{R}^{3 n}\right), \alpha>0$ is generated by the wavefunctions $f$ (with compact support in momentum space) for which one can find an approximating sequence $f_{s}, s \geqq 1^{5}$ with the properties:

i) $\tilde{f}_{s}\left(\boldsymbol{p}_{1} \ldots \boldsymbol{p}_{n}\right)$ has (for all $s \geqq 1$ ) its support within a fixed compact region of $\mathbb{R}^{3 n}$.

ii) $\left\|f_{s}^{(j)}\right\| \leqq c_{|j|}\left(1+s^{|j|}\right)$ for all multi-indizes $(j)$.

iii) $\left\|f-f_{s}\right\| \leqq c \cdot s^{-\alpha}$.

The set of those functions which are not contained in any of the spaces $M_{\alpha}\left(\mathbb{R}^{3 n}\right), \alpha>0$ will be denoted by $M_{0}\left(\mathbb{R}^{3 n}\right)^{6}$.

The second step is to remove the threshold contributions from $f_{s}$. To this end we take a function $h \in \mathscr{C}^{\infty}\left(\mathbb{R}^{3}\right) ; \grave{h}(\boldsymbol{u})=0$ for $|\boldsymbol{u}| \leqq 1$ and $\grave{h}(\boldsymbol{u})=1$ for $|\boldsymbol{u}| \geqq 2$. This function will be used to construct the smooth approximations $f_{r, s}$ with non-overlapping momenta:

$$
\tilde{f}_{r, s}\left(\boldsymbol{p}_{1} \ldots \boldsymbol{p}_{n}\right)=\prod_{m>m^{\prime}} \stackrel{\circ}{h}\left(r\left[\boldsymbol{p}_{m}-\boldsymbol{p}_{m^{\prime}}\right]\right) \cdot \tilde{f}_{s}\left(\boldsymbol{p}_{1} \ldots \boldsymbol{p}_{n}\right), \quad r \geqq 1 .
$$

It follows from the support properties of $(1-h)$ and the smoothness of $f_{s}$ and $h$ in momentum space that $\left\|f-f_{r, s}\right\| \leqq\left\|f-f_{s}\right\|+c \cdot\left(\frac{s}{r}\right)^{3 / 2}$, $c$ being independent of $r$ and $s$. A straightforward calculation also shows that $\left\|f_{r, s}\right\|_{N} \leqq c_{N} \cdot r^{N} \cdot(r+s)^{N}$ for $r, s \geqq 1$; the constant $c_{N}$ again does not depend on $r$ and $s$.

Now we replace $g$ in relation (4) by the 2-parameter family $f_{r, s}$. The following inequality which holds for arbitrary $N \in \mathbb{N}, N>1$ is

${ }^{5}$ The explicit construction of $f_{s}$ given above need not be optimal.

6 To illustrate this somewhat technical characterisation of the wavefunctions let us give an example: $f$ is an element of $M_{\alpha}\left(\mathbb{R}^{3 n}\right)$ if the configuration space integral $\int \prod_{l=1}^{n} d^{3} x_{l}\left|\left(\sum_{l^{\prime}=1}^{n}\left|\boldsymbol{x}_{l^{\prime}}\right|\right)^{\alpha} f\left(\boldsymbol{x}_{1} \ldots \boldsymbol{x}_{n}\right)\right|^{2}$ exists. 
then a consequence of the estimates just given:

$$
\left\|\Psi_{f}^{\text {out }}-\Psi_{f}(t)\right\| \leqq c_{N} \cdot r^{N} \cdot(r+s)^{N} \cdot t^{-N+1}+c \cdot\left\|f-f_{s}\right\|+c^{\prime} \cdot\left(\frac{s}{r}\right)^{3 / 2}
$$

with constants $c_{N}, c, c^{\prime}$ not depending on $r, s$ or $t$. It is easy to minimize the right hand side of this inequality with respect to $r$ and $s$ and one obtains:

Corollary. i) Let $f$ be an element of $M_{\alpha}\left(\mathbb{R}^{3 n}\right), \alpha>0$. Then for any positive $\gamma<\frac{3 \alpha}{6+4 \alpha}$ there exists a constant $c_{\gamma}$ such that

$$
\left\|\Psi_{f}^{\text {out }}-\Psi_{f}(t)\right\| \leqq c_{\gamma} \cdot t^{-\gamma} \quad \text { for } \quad t>0 .
$$

ii) For $f \in M_{0}\left(\mathbb{R}^{3 n}\right)$ and $\gamma<\frac{1}{2}$ we get the estimate

$$
\left\|\Psi_{f}^{\text {out }}-\Psi_{f}(t)\right\| \leqq c_{\gamma} \cdot d\left(t^{\gamma}\right) \text { for large } t .
$$

(Here we have introduced the distance function $d(s)=\left\|f-f_{s}\right\|$.)

Loosely speaking, $\left\|\Psi_{f}^{\text {out }}-\Psi_{f}(t)\right\|$ approaches 0 almost like $t^{-3 \alpha /(6+4 \alpha)}$ for $\alpha<\infty$ and like $t^{-3 / 4}$ for $\alpha=\infty$. It is the threshold contributions that prevent the norm distances $\left\|\Psi_{f}^{\text {out }}-\Psi_{f}(t)\right\|$ from decreasing faster than $t^{-3 / 4}$, even for smooth wavefunctions $f$. For wavefunctions vanishing at the thresholds $\boldsymbol{p}_{i}=\boldsymbol{p}_{j}, i \neq j$ one can get better estimates (depending on the order of the zeros and $\alpha$ ) for the rate of convergence of the HaagRuelle approximations $\Psi_{f}(t)$. Since the argument is the same as above we leave the details to the reader.

Now we come to the postponed proof of the theorem. We start with a trivial lemma clarifying the connection between the difference of the momenta $(\boldsymbol{p}-\boldsymbol{q})$ and the difference of the corresponding velocities $\left(\frac{p}{\omega_{p}}-\frac{q}{\omega_{q}}\right):$

Lemma 1. i) The $3 \times 3$-matrix $R(\boldsymbol{p}, \boldsymbol{q})$ defined by

$$
R(\boldsymbol{p}, \boldsymbol{q})_{i k}=2 \frac{\omega_{\boldsymbol{p}} \omega_{\boldsymbol{q}}}{\omega_{\boldsymbol{p}}+\omega_{\boldsymbol{q}}} \cdot\left\{\delta_{i k}+\frac{(\boldsymbol{p}+\boldsymbol{q})_{i} \cdot(\boldsymbol{p}+\boldsymbol{q})_{k}}{\left(\omega_{\boldsymbol{p}}+\omega_{\boldsymbol{q}}\right)^{2}-|\boldsymbol{p}+\boldsymbol{q}|^{2}}\right\} \quad i, k=1,2,3
$$

transforms $\left(\frac{\boldsymbol{p}}{\omega_{\boldsymbol{p}}}-\frac{\boldsymbol{q}}{\omega_{\boldsymbol{q}}}\right)$ into $(\boldsymbol{p}-\boldsymbol{q})$.

ii) The components of this matrix are arbitrarily often differentiable with respect to $\boldsymbol{p}$ and $\mathbf{q}$ and the derivatives are bounded for all (j) according to

$$
\left|R^{(j)}(\boldsymbol{p}, \boldsymbol{q})_{i k}\right| \leqq c_{|j|} \cdot(1+|\boldsymbol{p}|+|\boldsymbol{q}|)^{3} .
$$

(The proof of this statement is trivial and can be omitted.) We need the matrix $R(\boldsymbol{p}, \boldsymbol{q})$ to construct some operators which will occur later in 
our estimates. These operators are defined on the functions $g \in \mathscr{\mathscr { S }}\left(\mathbb{R}^{3 n}\right)$ : let $a \neq b$ be any two elements of the index set $\{1 \ldots n\}$, then

and

$$
\left.\widetilde{\left(\boldsymbol{S}_{a b} g\right.}\right)\left(\boldsymbol{p}_{1} \ldots \boldsymbol{p}_{n}\right)=R\left(\boldsymbol{p}_{a}, \boldsymbol{p}_{b}\right) \cdot \frac{\left(\boldsymbol{p}_{a}-\boldsymbol{p}_{b}\right)}{\left|\boldsymbol{p}_{a}-\boldsymbol{p}_{b}\right|^{2}} \tilde{g}\left(\boldsymbol{p}_{1} \ldots \boldsymbol{p}_{n}\right)
$$

$$
\left(\widetilde{T_{a b} g}\right)\left(\boldsymbol{p}_{1} \ldots \boldsymbol{p}_{n}\right)=\left(\nabla_{a}-\nabla_{b}\right) \cdot\left(\widetilde{\boldsymbol{S}_{a b} g}\right)\left(\boldsymbol{p}_{1} \ldots \boldsymbol{p}_{n}\right) .
$$

It is obvious that the function $\left(T_{a b} g\right)$ and the components of the vectorvalued function $\left(\boldsymbol{S}_{a b} g\right)$ are again elements of $\mathscr{\mathscr { S }}\left(\mathbb{R}^{3 n}\right)$. Since the definition of the norms $\|.\|_{N}$ has been adjusted to the properties of the operators $S_{a b}$ and $T_{a b}$ one can easily verify the following proposition:

Lemma 2. Let $g$ be an element of $\mathscr{\mathscr { S }}\left(\mathbb{R}^{3 n}\right)$. Then there exist for all $N \in\{0, \mathbb{N}\}$ certain constants $c_{N}$ and $c_{N}^{\prime}$ (not depending on $g$ ) such that $\left\|\left(S_{a b} g\right)_{l}\right\|_{N} \leqq c_{N} \cdot\|g\|_{N+1} \quad$ for $\quad l=1,2,3$ and $\left\|\left(T_{a b} g\right)\right\|_{N} \leqq c_{N}^{\prime} \cdot\|g\|_{N+1}$. (Here $\|.\|_{0}$ stands for the $L^{2}\left(\mathbb{R}^{3 n}\right)$ norm $\left.\|\|.\right)$

In the next lemma an important estimate is given for the asymptotic behaviour of the configuration-space wavefunction $g\left(t \mid x_{1} \ldots x_{n}\right)^{7}$ at large times $t$.

Lemma 3. Let $g$ be an element of $\mathscr{S}\left(\mathbb{R}^{3 n}\right)$. Then for two arbitrary elements $a \neq b$ from the index set $\{1 \ldots n\}$ and any $N \in\{0, \mathbb{N}\}$ the following inequality holds:

$$
\left|t^{N} g\left(t \mid \boldsymbol{x}_{1} \ldots \boldsymbol{x}_{n}\right)\right| \leqq\left(1+\left|\boldsymbol{x}_{a}-\boldsymbol{x}_{b}\right|\right)^{N} \cdot g_{N, t}\left(\boldsymbol{x}_{1} \ldots \boldsymbol{x}_{n}\right) .
$$

The function $g_{N, t}\left(\boldsymbol{x}_{1} \ldots \boldsymbol{x}_{n}\right)$ is square-integrable and

$$
\left\|g_{N, t}\right\| \leqq c_{N} \cdot\|g\|_{N}
$$

with a constant $c_{N}$ not depending on $g$ and $t$.

Proof. Since

$$
\left(\nabla_{a}-\nabla_{b}\right) e^{-i t \sum_{l=1}^{n} \omega_{l}}=(-i t) \cdot\left(\frac{p_{a}}{\omega_{a}}-\frac{p_{b}}{\omega_{b}}\right) \cdot e^{-i t \sum_{l=1}^{n} \omega_{l}}
$$

one gets immediately after partial integration:

$$
i t \cdot g\left(t \mid \boldsymbol{x}_{1} \ldots \boldsymbol{x}_{n}\right)=\int \prod_{l=1}^{n} d^{3} p_{l} e^{-i t \omega_{l}} \cdot\left(\widetilde{T_{a b} e_{\boldsymbol{x}_{1} \ldots \boldsymbol{x}_{n}} \cdot g}\right)\left(\boldsymbol{p}_{1} \ldots \boldsymbol{p}_{n}\right) \text {. }
$$

Here we have introduced the function $e_{\boldsymbol{x}_{1} \ldots \boldsymbol{x}_{n}}$,

$$
\widetilde{e_{\boldsymbol{x}_{1} \ldots \boldsymbol{x}_{n}}}\left(\boldsymbol{p}_{1} \ldots \boldsymbol{p}_{n}\right)=(2 \pi)^{-3 n / 2} \cdot e^{i \sum_{l=1}^{n} \boldsymbol{x}_{l} \boldsymbol{p}_{l}} .
$$

\footnotetext{
7 See relation (2) of the preceding chapter.
} 
It follows from the definition of the operators $S_{a b}$ and $T_{a b}$ that

and therefore

$$
\begin{aligned}
\widetilde{\left(T_{a b} e_{x_{1} \ldots x_{n}} \cdot g\right)}\left(p_{1} \ldots p_{n}\right)= & i\left(\boldsymbol{x}_{a}-\boldsymbol{x}_{b}\right) \cdot\left(\widetilde{e_{x_{1} \ldots x_{n}} \cdot S_{a b} g}\right)\left(p_{1} \ldots p_{n}\right) \\
& +\left(\widetilde{e_{x_{1} \ldots x_{n}} \cdot T_{a b} g}\right)\left(p_{1} \ldots p_{n}\right)
\end{aligned}
$$

(a) $i t \cdot g\left(t \mid x_{1} \ldots x_{n}\right)=i\left(x_{a}-x_{b}\right) \cdot\left(S_{a b} g\right)\left(t \mid x_{1} \ldots x_{n}\right)+\left(T_{a b} g\right)\left(t \mid x_{1} \ldots x_{n}\right)$.

Now we can prove the lemma by induction. Since

$$
\int \prod_{l=1}^{n} d^{3} x_{l} \cdot\left|g\left(t \mid x_{1} \ldots x_{n}\right)\right|^{2}=\|g\|^{2}
$$

the statement is true for $N=0$. Let us therefore assume that the lemma holds for $N$. It follows from relation (a) that

$$
\begin{aligned}
\left|t^{N+1} g\left(t \mid x_{1} \ldots x_{n}\right)\right| \leqq 2\left(1+\left|x_{a}-x_{b}\right|\right) & \\
& \cdot\left(\sum_{l=1}^{3}\left|t^{N}\left(S_{a b} g\right)_{l}\left(t \mid x_{1} \ldots x_{n}\right)\right|+\left|t^{N}\left(T_{a b} g\right)\left(t \mid x_{1} \ldots x_{n}\right)\right|\right) .
\end{aligned}
$$

According to our assumption we get

$$
\begin{array}{r}
\left|t^{N}\left(S_{a b} g\right)_{l}\left(t \mid x_{1} \ldots x_{n}\right)\right| \leqq\left(1+\left|x_{a}-x_{b}\right|\right)^{N} \hat{g}_{l, N, t}\left(x_{1} \ldots x_{n}\right) \\
\text { with }\left\|\hat{g}_{l, N, t}\right\| \leqq c_{N} \cdot\left\|\left(S_{a b} g\right)_{l}\right\|_{N}
\end{array}
$$

$$
\begin{array}{r}
\left|t^{N}\left(T_{a b} g\right)\left(t \mid \boldsymbol{x}_{1} \ldots \boldsymbol{x}_{n}\right)\right| \leqq\left(1+\left|\boldsymbol{x}_{a}-\boldsymbol{x}_{b}\right|\right)^{N} \hat{g}_{N, t}\left(\boldsymbol{x}_{1} \ldots \boldsymbol{x}_{n}\right) \\
\text { with }\left\|\hat{g}_{N, t}\right\| \leqq c_{N} \cdot\left\|\left(T_{a b} g\right)\right\|_{N} .
\end{array}
$$

But we know from Lemma 2 that $\left\|\left(\boldsymbol{S}_{a b} g\right)_{l}\right\|_{N} \leqq c_{N}^{\prime} \cdot\|g\|_{N+1}$ and $\left\|T_{a b} g\right\|_{N}$ $\leqq c_{N}^{\prime \prime} \cdot\|g\|_{N+1}$ and this proves our statement.

Finally, some additional remarks about the 1-particle creation operators are necessary: if $A$ is an element from the set $\mathscr{P}$ one can define another almost local operator $j$ by

$$
j=(2 \pi)^{-2} \cdot \int d^{4} p i\left(p_{0}-\omega_{p}\right) \tilde{A}(p) .
$$

Since $A$ creates a 1-particle state from the vacuum it is obvious that $j \Omega=0$. From the fact that $\tilde{A}(p)$ has compact support it follows that $j$ is a bounded operator. Thus one can easily deduce from the decrease properties of the truncated vacuum expectation values of the operators $A$ and $j$ the following lemma:

Lemma 4. Let $A$ be any operator from the set $\mathscr{P}$ and $j$ the operator defined above. If $f$ is an arbitrary element from $L^{2}\left(\mathbb{R}^{3 n}\right)$, then

$$
\left\|\int \prod_{l=1}^{n} d^{3} x_{l} f\left(\boldsymbol{x}_{1} \ldots \boldsymbol{x}_{n}\right) A\left(\boldsymbol{x}_{1}\right) \ldots A\left(\boldsymbol{x}_{n}\right) \Omega\right\|^{2} \leqq c \cdot \int \prod_{l=1}^{n} d^{3} x_{l}\left|f\left(\boldsymbol{x}_{1} \ldots \boldsymbol{x}_{n}\right)\right|^{2}
$$


and

$$
\begin{array}{r}
\left\|\int \prod_{l=1}^{n} d^{3} x_{l} f\left(\boldsymbol{x}_{1} \ldots \boldsymbol{x}_{n}\right) A\left(\boldsymbol{x}_{1}\right) \ldots j\left(\boldsymbol{x}_{a}\right) \ldots A\left(\boldsymbol{x}_{n}\right) \Omega\right\|^{2} \\
\leqq c_{N} \cdot \sum_{b=a+1}^{n} \int \prod_{l=1}^{n} d^{3} x_{l}\left(1+\left|\boldsymbol{x}_{a}-\boldsymbol{x}_{b}\right|\right)^{-2 N} \cdot\left|f\left(\boldsymbol{x}_{1} \ldots \boldsymbol{x}_{n}\right)\right|^{2}
\end{array}
$$

for all $N \in \mathbb{N}$. The constants $c$ and $c_{N}$ in these inequalities do not depend on $f$.

Now we are almost finished: let $f$ be any wavefunction with compact support in momentum space and $g$ an element of $\mathscr{S}\left(\mathbb{R}^{3 n}\right)$. Then one gets for the norm distance $\left\|\Psi_{f}\left(t^{\prime \prime}\right)-\Psi_{f}\left(t^{\prime}\right)\right\|, t^{\prime \prime} \geqq t^{\prime}>0$ the estimate:

$$
\left\|\Psi_{f}\left(t^{\prime \prime}\right)-\Psi_{f}\left(t^{\prime}\right)\right\| \leqq\left\|\Psi_{g}\left(t^{\prime \prime}\right)-\Psi_{g}\left(t^{\prime}\right)\right\|+\left\|\Psi_{f-g}\left(t^{\prime \prime}\right)\right\|+\left\|\Psi_{f-g}\left(t^{\prime}\right)\right\| .
$$

It follows from the definition of the Haag-Ruelle approximations and Lemma 4 that $\left\|\Psi_{f-g}\left(t^{\prime \prime}\right)\right\| \leqq c \cdot\|f-g\|$ and $\left\|\Psi_{f-g}\left(t^{\prime}\right)\right\| \leqq c\|f-g\|$.Clearly, $\left\|\Psi_{g}\left(t^{\prime \prime}\right)-\Psi_{g}\left(t^{\prime}\right)\right\| \leqq \int_{t^{\prime}}^{t^{\prime \prime}} d t\left\|\partial_{t} \Psi_{g}(t)\right\|$ and therefore one has only to consider the state $\partial_{t} \Psi_{g}(t)$. It is standard to prove the relation

$$
\partial_{t} \Psi_{g}(t)=\sum_{a=1}^{n} \int \prod_{l=1}^{n} d^{3} x_{l} g\left(t \mid \boldsymbol{x}_{1} \ldots \boldsymbol{x}_{n}\right) A\left(t, \boldsymbol{x}_{1}\right) \ldots j\left(t, \boldsymbol{x}_{a}\right) \ldots A\left(t, \boldsymbol{x}_{n}\right) \Omega,
$$

$j$ being the operator defined above. Because of the translation invariance of the vacuum and Lemma 4 one concludes that for all $N \in \mathbb{N}$

$$
\left\|\partial_{t} \Psi_{g}(t)\right\|^{2} \leqq c_{N}^{\prime \prime} \sum_{a=1}^{n} \sum_{b=a+1}^{n} \int \prod_{l=1}^{n} d^{3} x_{l}\left(1+\left|\boldsymbol{x}_{a}-\boldsymbol{x}_{b}\right|\right)^{-2 N}\left|g\left(t \mid \boldsymbol{x}_{1} \ldots \boldsymbol{x}_{n}\right)\right|^{2}
$$

Applying Lemma 3 to the right hand side of this inequality one gets

$$
\left\|\partial_{t} \Psi_{g}(t)\right\| \leqq c_{N}^{\prime} \cdot\|g\|_{N} \cdot t^{-N}
$$

with a constant $c_{N}^{\prime}$ not depending on $g$. This shows (after integration) that

$$
\left\|\Psi_{f}\left(t^{\prime \prime}\right)-\Psi_{f}\left(t^{\prime}\right)\right\| \leqq c_{N} \cdot\|g\|_{N} \cdot\left(\left(t^{\prime \prime}\right)^{-N+1}+\left(t^{\prime}\right)^{-N+1}\right)+c\|f-g\|
$$

for all $N \in \mathbb{N}, N>1$. If one now puts $t^{\prime \prime}=\infty$ and $t^{\prime}=t$ it follows

$$
\left\|\Psi_{f}^{\text {out }}-\Psi_{f}(t)\right\| \leqq c_{N} \cdot\|g\|_{N} \cdot t^{-N+1}+c \cdot\|f-g\|
$$

and this, finally, proves the theorem.

Acknowledgements. I am indebted to Professor R. Haag for his constant interest and encouragement and to V. Enß and Dr. M. Rinke for many helpful comments. I also wish to thank the DAAD for financial support. 


\section{References}

1. Haag, R.: Phys. Rev. 112, 669 (1958)

2. Ruelle, D.: Helv. Phys. Acta 35, 147 (1962)

3. Araki, H., Haag, R.: Commun. math. Phys. 4, 77 (1967)

4. Araki, H.: Lecture Notes, Zürich (1967)

5. Hepp, K.: Brandeis University Summer Institute in Theoretical Physics 1, 137 (1965)

6. Jost, R.: The general theory of quantized fields. Providence: Amer. Math. Soc. (1965) Streater, R.F., Wightman, A.S.: PCT, Spin and Statistics. New York: W. A. Benjamin 1964

7. Epstein, H.: J. Math. Phys. 8, 750 (1967)

Communicated by R. Haag

D. Buchholz

II. Institut für Theoretische Physik

der Universität

D-2000 Hamburg 50, Luruper Chaussee 149

Federal Republic of Germany 
